\title{
CLASSIFICATION OF FIRST ORDER THEORIES WHICH HAVE A STRUCTURE THEOREM
}

\author{
BY SAHARON SHELAH ${ }^{1}$
}

We first explain the problem, then the solution and various consequences; we then discuss the limits and possible criticisms of our solution. Full proofs will appear in [8].

Let $T$ denote a countable complete first order theory. A model $M$ of $T$ is a set $|M|$ with interpretations of the predicates and the function symbols appearing in $T$ as relations and functions on $|M|$.

1. The problem. As we view model theory also as an abstract algebra (i.e., dealing with any $T$, not just a specific one), we want to find a general structure theorem for the class of models of $T$ like those of Steinitz (for algebraically closed fields) and Ulm (for countable torsion abelian groups). So, ideally, for every model $M$ of $T$ we should be able to find a set of invariants which is complete, i.e., determines $M$ up to isomorphism. Such an invariant is the isomorphism type, so we should better restrict ourselves to more reasonable ones, and the natural candidates are cardinal invariants or reasonable generalizations of them. For a vector space over $Q$ we need one cardinal (the dimension); for a vector space over an algebraically closed field, two cardinals; for a divisible abelian group $G$, countably many cardinals (the dimension of $\{x \in G: p x=0\}$ for each prime $p$ and the rank of $G / \operatorname{Tor}(G))$; and for a structure with countably many one-place relations (i.e. $\equiv$ distinguished subsets), we need $2^{\aleph_{0}}$ cardinals (the cardinality of each Boolean combination).

We believe the reader will agree that every model $(|M|, E)$, where $E$ is an equivalence relation, has a reasonably complete set of invariants: namely, the function saying, for each cardinal $\lambda$, how many equivalence classes of this power occur. Also, if we enrich $M$ by additional relations which relate only equivalent members and such that each equivalence class becomes a model with a complete set of invariants, then the resulting model will have a complete set of invariants.

However, even if we allow such generalized cardinal invariants, we cannot have such a structure theory for every $T$, so we have to reformulate our problem.

1.1. The Structure/Nonstructure Problem. Describe for some $T$ 's a structure theory and prove for the other theories nonstructure theorems showing that no structure theory is possible.

Received by the editors February 22, 1984.

1980 Mathematics Subject Classification. Primary 03C45.

Key words and phrases. Structure theorem, Morley's conjecture, spectrum problem, stable, superstable, dop, otop.

${ }^{1}$ The author would like to thank the BSF (United States-Israel Binational Science Foundation), the NSF and the NSERC of Canada for partially supporting this research. 
Let us try to explicate this matter. We define a $\lambda$-value of depth $\alpha$ : for $\alpha=0$ it is a cardinal $\leq \lambda$, for $\alpha=\beta+1$ it is a sequence of length $\leq 2^{\aleph_{0}}$ of functions from the set of $\lambda$-values of depth $\beta$ to the set of cardinals $\leq \lambda$ or a $\lambda$-value of depth $\beta$, and for $\alpha$ a limit ordinal it is a $\lambda$-value of depth $<\alpha$.

An invariant [of depth $\alpha$ ] for models of $T$ is a function giving, for every model of $M$ of $T$ of power $\lambda$, some $\lambda$-value [of depth $\alpha$ ] which depends only on the isomorphism type of $M$. If we do not restrict $\alpha$, the set of possible values of the invariants is known to be as complicated as the set of all models. This leads to:

1.2. THESIS. A theory $T$ has a structure theory if there are an ordinal $\alpha$ and invariants (or sets of invariants) of depth $\alpha$ which determine every model (of T) up to isomorphism.

We can prove easily, by induction on the ordinal $\alpha$, that the number of $\aleph_{\gamma}$-values of depth $\alpha$ has a bound $\beth_{\alpha}(|\omega+\gamma|)$ where

$$
\beth_{\beta}(\mu)=\mu+\sum_{\gamma<\beta} 2^{\beth_{\gamma}(\mu)} .
$$

1.3. COROLlARY OF THE THESIS. If T has a structure theory then there is an $\alpha$ such that for every $\gamma, T$ has $\leq \beth_{\alpha}(|\omega+\gamma|)$ nonisomorphic models of power $\aleph_{\gamma}$.

It is easy to show (assuming, e.g., that G.C.H. holds) that for every $\alpha$ there are many $\gamma$ 's such that $\beth_{\alpha}(|\omega+\gamma|)<2^{\aleph_{\gamma}}$. Thus, if one is able to show that the theory has $2^{\aleph_{\gamma}}$ models of power $\aleph_{\gamma}$, this establishes nonstructure. So we are lead to a more concrete question: the spectrum problem, i.e., what can be the function $I$ given by $I(\lambda, T)=$ the number of nonisomorphic models of $T$ of power $\lambda$. We can hope that a nonstructure theorem should imply it is large, whereas a structure theorem should enable us to show it is small and even allow us to compute it.

It was our firm belief that there is such a theory, and that we should look at what occurs at large enough cardinals, as in small cardinals various "incidental" facts interfere. Notice that a priori there need not be a solution to the structure/nonstructure problem or to the spectrum problem: maybe $I(\lambda, T)$ can be any one of a family of complicated functions, or, worse, maybe we cannot charcterize reasonably those functions, or, worst of all, maybe the question of which functions occur is independent of the usual axioms of set theory.

\section{Solution.}

2.1. The MAIN TheOREM. For every $T$ either $I(\lambda, T)=2^{\lambda}$ for every uncountable $\lambda$, or $I\left(\aleph_{\alpha}, T\right)<\beth_{\omega_{1}}(|\alpha|)$; moreover, every model of $T$ can be characterized up to isomorphism by an invariant of countable depth.

To explain this, we need to consider a related problem, more general and vague, which is open-ended. 
2.2. The Classification Problem. Classify the $T$ 's in a useful way, i.e., such that for suitable questions on the class of models of $T$ the partition to cases according to the classification will be helpful.

Notice that " $T$ extends Peano arithmetic" or " $T$ extends ring theory" are totally uninteresting for what we seek. Although much has been proved about such theories, it seems we cannot say anything on theories failing to have these properties. We really look for pairs of properties which we can prove are complementary, and each has strong consequences. Note also that for each dichotomy we get something on the "simpler" side; though still not all of its members have a structure theory, they have "positive" properties interesting in their own right (like existence/uniqueness of prime models).

In fact, five such dichotomies, determined by the properties stable, superstable (see [3]), dop, deep (see [4]), and otop (see [8]), are relevant to the solution. Fortunately, we can explain those properties. $T$ is unstable if in some sense $T$ can define order: for some $n$ and (first order) formula $\varphi$ for every order $I$ there are a model $M$ of $T$ and sequences $\bar{a}_{t}(t \in I)$ of length $n$ of elements of $M$ such that in $M, \varphi\left(\bar{a}_{t}, \bar{a}_{s}\right)$ holds iff $t<s$ (in $I$ ).

Now we restrict our discussion to stable theories, and here we shall be somewhat vague. A theory is unsuperstable if we can define in it a tree with $\omega+1$ levels (by $\omega$ formulas); a theory has the dop (dimensional order property) if we can define order as above, not by a first-order formula, but by the question "is some dimension related to $\bar{a}_{s}, \bar{a}_{t}$ uncountable?" Next, a theory has the otop (omitting type order property) if there is a sequence $\left\langle\varphi_{m} \mid m<\omega\right\rangle$ of formulas such that for every order $I$ there are a model $M$ and $n$-tuples $\bar{a}_{t}(t \in I)$ of members of $M$ such that $s<t$ iff there is a $k$-tuple $\bar{c}$ of members of $M$ such that $\varphi_{m}\left(\bar{c}, \bar{a}_{s}, \bar{a}_{t}\right)$ holds for every $m$. The property complementary to the otop (for a superstable theory) is having prime models over quite a number of sets (see below).

2.3. THEOREM. If $T$ is unstable, or stable but not superstable, or has the dop or the otop, then for uncountable $\lambda, I(\lambda, T)=2^{\lambda}$.

There are other facts indicating that such $T$ 's have nonstructure properties (for logicians: for regular $\lambda>\aleph_{0}$ such $T$ have nonisomorphic models $M$ of power $\lambda$ which can be made isomorphic by $\lambda$-complete forcing. Also, for each regular $\lambda>\aleph_{0}$ there is a function $s$ on the set of the isomorphism types of the models of power $\lambda$ of $T$ such that its range is the set of all subsets of $\left\{\delta<\lambda: \operatorname{cf} \delta=\aleph_{0}\right\}$ modulo the club filter. These facts indicate strong nonabsoluteness of the isomorphism type). For all other theories $T$ we shall establish a decomposition theorem which implies that the phenomena just mentioned do not occur for the theories and $\lambda>\beth_{1}$.

2.4. Generic examples. To illustrate the properties we give examples, "forgetting" the "completeness". Among unstable theories, consider the theories of linear order and of graphs (the formula witnessing that the latter is unstable is $\phi\left(\left\langle x_{0}, x_{1}\right\rangle,\left\langle y_{0}, y_{1}\right\rangle\right)=$ " $x_{0}, y_{1}$ are connected"). Among stable nonsuperstable theories consider the theory of $\left({ }^{\omega} \omega, \ldots, E_{n}, \ldots\right)$, where ${ }^{\omega} \omega$ is the set of functions from the natural numbers to the natural numbers, and $E_{n}$ is the equivalence relation $f E_{n} g$ iff $(\forall m<n) f(m)=g(m)$; another example is 
the class of abelian groups (use the formulas $x E_{n} y$ if $p^{n} \mid(x-y)$ for a fixed $p$ ). For superstable $T$ with the dop, consider the theory of the model $M$ with universe

$$
\omega_{1} \cup\left\{\langle\alpha, \beta, \gamma\rangle: \alpha \neq \beta<\omega, \gamma<\omega_{1}, \text { and }[\alpha<\beta \Rightarrow \gamma<\omega]\right\},
$$

relation $P=\omega_{1}$, and functions $F_{1}, F_{2}: F_{1}(\alpha)=\alpha, F_{2}(\alpha)=\alpha, F_{1}(\langle\alpha, \beta, \gamma\rangle)=$ $\alpha, F_{2}(\langle\alpha, \beta, \gamma\rangle)=\beta$ (so we can define an order on $P$ by "the cardinality of $\left\{x: F_{1}(x)=a, F_{2}(x)=b\right\}$ is countable").

An example of a superstable $T$ with the optop but not the dop is a little complicated so we omit it.

2.5. Superstable without the dop and otop (i.e., exactly the theories not covered by Theorem 2.3). For any such $T$ there is a kind of structure theorem:

The Decomposition Theorem. For any model $M$ of $T$ there is a tree $I$ with $\omega$ levels (e.g., a set of finite sequences of ordinals) and submodels $N_{t}(t \in I)$ such that $\eta<\nu \Rightarrow N_{\eta} \subset N_{\nu}, N_{\eta}$ has power $\leq 2^{\aleph_{0}}$, each $N_{\eta}$ is an elementary submodel of $M$, the tree is "free" [technically, nonforking; i.e., for every $\eta=\nu \frown\langle\alpha\rangle$, if $\bar{c} \in N_{\eta}$ then its type over $\bigcup\left\{N_{\tau}: \eta \not z\right\}$ is finitely satisfiable in $N_{\nu}$ ] and $M$ is prime and minimal over $\bigcup_{\eta \in I} N_{\eta}$.

This tree of models determines the model up to isomorphism (but as the tree is not unique it is not the invariant we seek). We still have to ask if the tree has an infinite branch. If this may occur, $T$ is called deep. A canonical example: the theory of one unary function is deep. Otherwise there is a monotonically decreasing function from $I$ to a countable ordinal $\mathrm{Dp}(T)$. The reader may guess that we have now arrived at $T$ with a structure theorem (but as the tree is not unique, we have no yet obtained the invariant). For example, we can count the number of possible $\left\langle N_{\eta}: \nu \leq \eta \in I\right\rangle$ over $N_{\nu}$ by downward induction on $\nu \in I$, proving that $I\left(\aleph_{\alpha}, T\right)<\beth_{\omega_{1}}(|\omega+\alpha|)$, which in many cases is smaller than $\aleph_{\alpha}$. On the other hand, for deep theories we have $I(\lambda, T)=2^{\lambda}$ for $\lambda$ uncountable.

An example of such a nondeep theory is that having a one-place function $F$ satisfying $F^{n+1}(x)=F^{n}(x)$. For this theory $I\left(\aleph_{\gamma}, T\right)$ is $\leq \beth_{n-1}(|\gamma+\omega|)$, where $n \geq 2$.

3. Consequences. We try to indicate here that many problems in this direction can now be solved.

3.1. Morley's conjecture and the spectrum problem. The relevant test question for structure theory was Morley's conjecture, which says that $\lambda \leq \mu \Rightarrow$ $I(\lambda, T) \leq I(\mu, T)$ except when $\lambda=\aleph_{0}<\mu, T$ categorical in $\aleph_{1}$, but not in $\aleph_{0}$. The classification enables us to prove this conjecture. As for the spectrum, if $T$ is unsuperstable or has the dop or otop, $I(\lambda T)=2^{\lambda}$ for $\lambda>\aleph_{0}$, and this also holds for a deep $T$. In the remaining case, if $\operatorname{Dp}(T) \geq \omega, \alpha>0$, then $I\left(\aleph_{\alpha}, T\right)=\beth_{\mathrm{Dp}(T)}(|\omega+\alpha|)$. If $\operatorname{Dp}(T)<\omega, \alpha \geq 2^{\aleph_{0}}$, then $I\left(\aleph_{\alpha}, T\right)=$ $\beth_{\mathrm{Dp}(T)-1}\left(|\alpha|^{\kappa}\right)$ for some fixed $1 \leq \kappa \leq 2^{\aleph_{0}}$ or $I\left(\aleph_{\alpha}, T\right)=\beth_{k}\left[\sum_{\lambda<\kappa} \beth_{l}\left(|\alpha|^{\lambda}\right)\right]$, where $l$ and $k$ are natural numbers associated with $T$, and $\kappa$ is some fixed cardinal $\leq\left(2^{\aleph_{0}}\right)^{+}$. We hope that only the first case occurs and $\kappa \in\left\{1, \aleph_{0}, 2^{\aleph_{0}}\right\}$ (this is a "continuum hypothesis" problem), and that the computation can 
be done for $\alpha<2^{\aleph_{0}}$ too, but though we know some people believe this is a major missing point, we could not make ourselves excited about it. It is, and had been, our opinion that the main point is the "main gap theorem", i.e., establishing that $I(\lambda, T)=2^{\lambda}$ or $I\left(\aleph_{\alpha}, T\right)<\beth_{\omega_{1}}(|\alpha|)$.

3.2. On the $L_{\infty, \lambda}$-equivalence. Now we return to the invariant. In the mid 1970s several people looked at the problem "for which $T$ are any two $L_{\infty, \lambda}$-equivalent models of power $\leq \lambda$ isomorphic". $\left(L_{\infty, \lambda}\right.$ is an infinitary logic in which we allow conjunction of any length, not necessarily finite, and $\left(\exists x_{0}, \ldots, x_{i}, \ldots\right)_{\imath<\alpha}$ for $\alpha<\lambda$.) We restrict ourselves to regular $\lambda>\beth_{1}$. In the cases where the answer to this problem is positive, the theory $T_{\infty, \lambda}$, in the logic $L_{\infty, \lambda}$, of a model can be viewed as a generalized invariant for the model. Therefore, a negative answer is a quite strong nonstructure theorem. We can prove that for $T$ unsuperstable or with the dop or otop, the answer is negative (for regular $\lambda>\beth_{1}$ ), and for the rest it is positive (so here deepness does not imply nonstructure in the present sense). The status of the theory $T_{\infty, \lambda}$ as an invariant can be viewed as dubious, since the sentences in it contain quantification of sequences of variables of any length $<\lambda$; but we cannot make do with quantifications of bounded length, since, for $\mu<\lambda, L_{\infty, \mu}$ does not distinguish between sets of power $\mu$ and sets of power $\mu^{+}$. However, we get similar results when we replace the logic $L_{\infty, \lambda}$ by the logic $L_{\lambda}^{*}$, which is $L_{\infty, \beth_{1}^{+}}$enriched by the addition of quantifiers which say that the dimension of a vector space (or even of a general dependence relation) is $>\mu$ for each $\mu<\lambda$. When we come to view the theories $T_{\lambda}^{*}$ in the logics $L_{\lambda}^{*}$ as invariants, we notice that the depth of the sentences in $T_{\lambda}^{*}$ is arbitrary (i.e., $<\lambda^{+}$), hence $T_{\lambda}^{*}$ is not an invariant of bounded depth. Now for nondeep theories, and only for them, we can restrict the $L_{\lambda}^{*}$-theories to sentences of countable depth.

3.3. The maximal number IE $(\lambda, T)$ of pairwise nonelementarily embeddable models of $T$ of power $\lambda$. This sounds like a variant of $I(\lambda, T)$, and we thought so until we tried to compute it for deep theories. Restricting ourselves to $\lambda$ regular $>2^{\aleph_{0}}$, we get the maximum number if $T$ is unsuperstable or has the dop or otop. Otherwise, if $T$ not deep, any family of pairwise nonelementarily embeddable models has power $<\beth_{\omega_{1}}$. In the remaining case $(T$ superstable without dop and otop but deep) there is a cardinal $\kappa$, not depending on $T$ [for logicians, it is the first beautiful cardinal, i.e., the first $\kappa \stackrel{\mathbf{w}}{\longrightarrow}(\omega), \omega$; it is strongly inaccessible but smaller than the first measurable and may exist in $L]$ such that for $\lambda<\kappa, I E(\lambda, T)=2^{\lambda}$, but for any models $M_{\imath}(i<\kappa)$ of $T$, for some $i<j, M_{\imath}$ is elementarily embeddable into $M_{\jmath}$.

3.4. Criticism and limits. (A) What about decidability of the theory? Just as biological taxonomy does not tell us whether a species is tasty, the classificaton here does not deal with decidability. Note that a theory with one equivalence relation may have a large Turing degree.

(B) Why not restrict yourself to universal theories or even varieties? In this case you can hope to get more explicit structure theorems. We think the first order case is the central one, and it is the traditional focus of model theory. In addition, the theory presented here seems the right way to solve those problems. If we define a universal theory $T$ (which is not necessarily complete) 
to be stable if every completion of it is stable, etc., then we get the same results as above. Moreover, in the decomposition theorem, $N$ is, in fact, generated by $\bigcup_{\eta \in I} N_{\eta}$ (and we can define "freeness" using only quantifier free formulas) (see [7]). Also, our approach has been followed in many investigations and results on specified classical theories.

(C) Why elementary classes? For example, chain conditions are not first order; so why not $\psi \in L_{\omega_{1} \omega}$ or pseudo-elementary classes? Surely it would be better to classify larger families. There are quite a few results on these (see $[\mathbf{5}$ and 3], resp.), but they run into problems independent of ZFC (and solving for first order seems to be a preliminary step).

(D) Why not make the structure theory be over a given structure? Why is linear order complicated? Why not let $T$ be uncountable? Those things seem to come naturally later, and there is some work on them.

(E) Is our classification incidental? We have seen some questions solved by it. There is considerable work on two others: the Keiser order [2] (coming from investigating ultrapowers-see $[3, \mathrm{VI}]$ ) and the strength of monadic logic on models of $T$ (see Baldwin and Shelah [1]). In the investigations of both, the classification presented here is widely used.

(F) Are there not additional properties missing? There are; the best known is "being totally transcendental" introduced by Morley. But by one of his examples there is a nontotally transcendental $T$ such that $I(\lambda, T)=$ $\operatorname{Min}\left\{2^{\lambda}, \beth_{2}\right\}$, so there are theories having the simplest possible invariants (short of being categorical) which are not totally transcendental, suggesting that the dichotomy t.t-non-t.t is not very useful. More interesting is the f.c.p. [introduced by Keisler [2], investigated in [3, II, §4]-see V]. Another property is the tree property (see [6]).

It surely would be interesting to refine the classification of unstable $T$. Moreover, the fact that such a general scheme was proved possible with a comprehensible solution, should encourage analogous work on continuations and analogous problems.

\section{REFERENCES}

1. J. Baldwin and S. Shelah, Classification of theories by second order quantifiers, Notre Dame J. Formal Logic, Proc. of the 1980/1981 Jerusalem model theory year.

2. J. Keisler, Ultraproducts which are not saturated, J. Symbolic Logic 32 (1967), 23-46.

3. S. Shelah, Classification theory, North-Holland, 1978.

4. _ The spectrum problem. I, $\aleph_{\varepsilon}$-saturated models, the main gap, Israel J. Math. 43 (1982), 324-356.

5. _ The classification of non-elementary classes. I, Israel J. Math. Part A, 46 (1983), 212-240; Part B 46 (1983), 241-243.

6. __ Simple unstable theories, Ann. Math. Logic 19 (1980), 177-204.

7. __ The spectrum problem. III: Universal theories, Israel J. Math.

8. _ C Classification theory - with solution for countable theories, rev. ed., North-Holland (to appear).

Department of Mathematics, The Hebrew University, Jerusalem, ISRAEL

Department of Mathematics, Simon Fraser University, Burnaby V5A 1S6, CANADA

Department of MAThematics, University of CAlifornia, Berkeley, CALIFORNIA 94720 